Research Article

\title{
A Novel Method for Developing Efficient Probability Distributions with Applications to Engineering and Life Science Data
}

\author{
Alamgir Khalil, ${ }^{1}$ Abdullah Ali H. Ahmadini $\left(\mathbb{D},{ }^{2}\right.$ Muhammad Ali, ${ }^{1}$ Wali Khan Mashwani $\left(\mathbb{D},{ }^{3}\right.$ \\ Shokrya S. Alshqaq, ${ }^{2}$ and Zabidin Salleh $\mathbb{1}^{4}$ \\ ${ }^{1}$ Department of Statistics, University of Peshawar, Peshawar, Khyber Pakhtunkhwa, Pakistan \\ ${ }^{2}$ Department of Mathematics, College of Science, Jazan University, Jazan, Saudi Arabia \\ ${ }^{3}$ Institute of Numerical Sciences, Kohat University of Science \& Technology, Kohat, Pakistan \\ ${ }^{4}$ Department of Mathematics, Faculty of Ocean Engineering Technology and Informatics, Universiti Malaysia Terengganu, \\ Kuala Nerus 21030, Terengganu, Malaysia
}

Correspondence should be addressed to Wali Khan Mashwani; mashwanigr8@gmail.com

Received 16 June 2021; Accepted 2 August 2021; Published 19 August 2021

Academic Editor: Zakia Hammouch

Copyright $\odot 2021$ Alamgir Khalil et al. This is an open access article distributed under the Creative Commons Attribution License, which permits unrestricted use, distribution, and reproduction in any medium, provided the original work is properly cited.

\begin{abstract}
In this paper, a new approach for deriving continuous probability distributions is developed by incorporating an extra parameter to the existing distributions. Frechet distribution is used as a submodel for an illustration to have a new continuous probability model, termed as modified Frechet (MF) distribution. Several important statistical properties such as moments, order statistics, quantile function, stress-strength parameter, mean residual life function, and mode have been derived for the proposed distribution. In order to estimate the parameters of MF distribution, the maximum likelihood estimation (MLE) method is used. To evaluate the performance of the proposed model, two real datasets are considered. Simulation studies have been carried out to investigate the performance of the parameters' estimates. The results based on the real datasets and simulation studies provide evidence of better performance of the suggested distribution.
\end{abstract}

\section{Introduction}

In the last few years, the literature of distribution theory has become rich due to the induction of additional parameters in the existing distribution. The inclusion of an extra parameter has shown greater flexibility compared to competitive models. The inclusion of a new parameter can be performed either using the available generator or by developing a new technique for generating new improved distribution compared to classical baseline distribution. Azzalini [1] proposed a modified form of the normal distribution by inserting an extra parameter, known as skew normal distribution, which indicated greater flexibility over normal distribution. Mudholkar and Srivastava [2] introduced exponentiated Weibull distribution by introducing a shape parameter in two-parameter Weibull distribution. Its cumulative distribution function is as follows:

$$
G(y ; \alpha, \lambda, \beta)=\left(1-e^{-\lambda x^{\alpha}}\right)^{\beta}, \quad x, \alpha, \lambda, \beta>0 .
$$

This model provides greater flexibility compared to the base line distribution. Note that, for $\beta=1$, the exponentiated Weibull distribution and base line distribution coincide. Later on, various researchers have introduced different forms of exponentiated distributions; see, for example, the work of Gupta et al. [3]. Marshall and Olkin [4] introduced another technique to add an extra parameter to a probability distribution. Eugene et al. [5] suggested the beta-generated technique and applied this method to beta distribution and proposed beta-generated distribution by incorporating an 
extra parameter in beta distribution. Alzaatreh et al. [6] proposed a new technique and produced $\mathrm{T}-\mathrm{X}$ class of continuous probability models by interchanging the probability density function of beta distribution with a probability density function, $g(t)$, of a continuous random variable and used a function $W(F(x))$ which fulfills some particular conditions. Recently, Aljarrah et al. [7] introduced $\mathrm{T}$-X class of distributions using quantile functions. For more details about new techniques to produce probability distributions, see the works of Lee et al. [8] and Jones [9]. AlAqtash et al. [10] proposed a new class of models using the logit function as a baseline and obtained the particular case referred to as Gumbel-Weibull distribution. Alzaatreh et al. [11] studied the gamma-X class of distributions and recommended the particular case using the normal distribution as a baseline distribution. Abid and Abdulrazak [12] introduced truncated Frechet-G class of distributions. Korkmaz and Genc [13] presented a generalized two-sided class of probability distributions. Alzaghal et al. [14] worked on the T-X class of distributions. Aldeni et al. [15] used the quantile function of generalized lambda distribution and introduced a new family. For more details, see the works of Cordeiro et al. [16], Alzaatreh et al. [17], and Nasir et al. [18]. The more recent modified Weibull distributions are introduced by Abid and Abdulrazak [12], Korkmaz and Genc [13], Aldeni et al. [15], Cordeiro et al. [16], and Pe and Jurek [19].

Pearson [20] used the system of differential equation technique and produced new probability distributions. Burr [21] also proposed a new method by using the differential equation method, which may take on a wide variety of forms of the continuous distributions. Since 1980, methodologies of suggesting new models moved to the inclusion of extra parameters to an existing family of distributions to increase the level of flexibility. These include Weibull-G presented by Bourguignon et al. [22], Garhy-G proposed by Elgarhy et al. [23], Kumaraswamy (Kw-G) proposed by Cordeiro and de Castro [24], Type II half-logistic-G by Hassan et al. [25], exponentiated extended-G suggested by Elgarhy et al. [26], the Kumaraswamy-Weibull introduce by Hassan and Elgarhy [27], exponentiated Weibull by Hassan and Elgarhy [28], odd Frechet-G introduced by Haq and Elgarhy [29], and Muth-G by Almarashi and Elgarhy [30]. For a short review, one can study the work of Kotz and Vicari [31]. Recently, Mahdavi and Kundu [32] developed a new technique for proposing probability distributions which is referred as alpha power transformation (APT) technique, defined by the cumulative distribution function (CDF) as follows:

$$
F_{\mathrm{APT}}(x)= \begin{cases}\frac{\alpha^{F(x)}-1}{\alpha-1}, & \text { if } \alpha>0, \alpha \neq 1, \\ F(x), & \text { if } \alpha=1 .\end{cases}
$$

The core purpose of introducing new family of distributions is to overcome the difficulties that are present in the existing probability distributions. In this study, we suggest a new method for obtaining new continuous probability distributions. Frechet distribution is used as a submodel to have a new probability distribution which is referred as modified Frechet (MF) distribution. Our proposed family of distributions also models monotonic and nonmonotonic hazard rate function and provides increased flexibility as compared to the already available probability distribution in the literature.

\section{The Proposed Class of Distributions}

The proposed class of probability distributions is termed as modified Frechet class (MFC) of distributions. The cumulative distribution function (CDF) and probability density function (PDF) of the suggested class of distributions are given by the following expressions:

$$
\begin{gathered}
G_{\mathrm{MFC}}(x)=\frac{e^{-(F(x))^{\alpha}}-1}{\left(e^{-1}-1\right)}, \quad x>0, \\
g_{\mathrm{MFC}}(x)=\frac{\alpha f(x)(F(x))^{\alpha-1} e^{-(F(x))^{\alpha}}}{\left(1-e^{-1}\right)}, \quad x>0,
\end{gathered}
$$

where $F(x)$ and $f(x)$ are the CDF and PDF of the baseline distribution and $\alpha$ is the shape parameter. The method in (3) is used to produce a new model referred as modified Frechet (MF) distribution with the aim to attain more flexibility in modeling life time data. The derivation of MF distribution is given in Section 2.1 .

2.1. The Proposed Distribution. The CDF of Frechet distribution is as follows:

$$
F(x)=e^{-x^{-\beta}}, \quad x>0
$$

where $\beta$ is the shape parameter.

This portion of the manuscript is concerned with introducing a subclass of MF class of distributions using the cumulative distribution function of Frechet distribution. The resultant distribution is what we call modified Frechet (MF) distribution.

Definition 1. A random variable $X$ is said to have MF distribution with two parameters $\alpha$ and $\beta$ if its PDF is given as follows:

$$
f_{\mathrm{MF}}(x)=\frac{\alpha \beta x^{-(\beta+1)} e^{-\alpha x^{-\beta}-e^{-\alpha x}-\beta}}{\left(1-e^{-1}\right)}, \quad x>0 .
$$

Its $\mathrm{CDF}$ is given by

$$
F_{\mathrm{MF}}(x)=\frac{\left.e^{-\left(e^{-\alpha x}-\beta\right.}\right)}{\left(e^{-1}-1\right)}, \quad x>0 .
$$

The hazard rate function of MF distribution is as follows:

$$
h_{\mathrm{MF}}(x)=\frac{\alpha \beta x^{-(\beta+1)} e^{-\alpha x^{-\beta}-e^{-\alpha x}-\beta}\left(e^{-1}-1\right)}{\left(e^{-1}-e^{-\left(e^{-\alpha x^{-\beta}}\right)}\right)\left(1-e^{-1}\right)} \text {. }
$$


The survival function of the MF model is given by

$$
S_{\mathrm{MF}}(x)=\frac{e^{-1}-e^{-\left(e^{-\alpha x^{-\beta}}\right)}}{e^{-1}-1} \text {. }
$$

These four functions have been plotted in Figures 1 and 2

Lemma 1. If $f(x)$ is decreasing function, then $f_{M F}(x)$ is also decreasing function for $0 \leq \alpha<1$ and $\beta>0$.

Proof. If $f(x)$ is a differentiable function and if $f^{\prime}(x)<0$ or $(\mathrm{d} / \mathrm{d} x) \ln f(x)<0$ for all $X$, then $f(x)$ is a decreasing function and vice versa.

Taking first derivative of $\ln f_{\mathrm{MF}}(x)$, we have

$$
\begin{aligned}
& \frac{\mathrm{d}}{\mathrm{d} x} \ln f_{\mathrm{MF}}(x)=\frac{\mathrm{d}}{\mathrm{d} x} \ln \left[\frac{\alpha \beta x^{-(\beta+1)} e^{-\alpha x^{-\beta}-e^{-\alpha x^{-\beta}}}}{\left(1-e^{-1}\right)}\right], \\
& \frac{\mathrm{d}}{\mathrm{d} x} \ln f_{\mathrm{MF}}(x)=-\frac{\beta}{x}-\frac{1}{x}-\alpha \beta x^{-\beta-1}\left(e^{-\alpha x^{-\beta}}-1\right) .
\end{aligned}
$$

Thus, for $0 \leq \alpha<1$ and $\beta>0,(\mathrm{~d} / \mathrm{d} x) \ln f_{\mathrm{MF}}(x)<0$. This concludes the lemma.

Lemma 2. For $\alpha<1$, if $f(x)$ is log-convex and decreasing function, then $h_{M F}(x)$ is a decreasing function.

Proof. If the second-order differential of $f(x)$ exists and $\left(\mathrm{d}^{2} / \mathrm{d} x^{2}\right) \ln f(x)>0$, then $f(x)$ is said to be log-convex. Taking second-order derivative of equation (10), we obtain

$$
\begin{aligned}
& \frac{\mathrm{d}^{2}}{\mathrm{~d} x^{2}} \ln f_{\mathrm{MF}}(x)=\frac{\mathrm{d}}{\mathrm{d} x}\left(-\frac{\beta}{x}-\frac{1}{x}-\alpha \beta x^{-\beta-1}\left(e^{-\alpha x^{-\beta}}-1\right)\right), \\
& \frac{\mathrm{d}^{2}}{\mathrm{~d} x^{2}} \ln f_{\mathrm{MF}}(x)=\frac{\beta}{x^{2}}+\frac{1}{x^{2}}+\alpha \beta x^{-\beta-2}\left[(-\beta-1)\left(e^{-\alpha x^{-\beta}}-1\right)+\alpha \beta x^{-\beta} e^{-\alpha x^{-\beta}}\right] .
\end{aligned}
$$

When $0 \leq \alpha<1$ and $\beta>0$, then $\left(\mathrm{d}^{2} / \mathrm{d} x^{2}\right) \ln f_{\mathrm{MF}}(x)>0$. Therefore, for $0<\alpha<1, f_{\mathrm{MF}}(x)$ is log-convex [33].

2.1.1. Quantile Function. Let $X \sim \operatorname{MF}(\alpha, \beta)$; then, the quantile function is as follows:

$$
F(X)=U \Longrightarrow X=F^{-1}(U)
$$

where $U$ is uniformly distributed random variable. The quantile function of the MF model is given as

$$
X_{p}=\left[-\frac{1}{\alpha} \ln \left\{-\ln \left(u\left(e^{-1}-1\right)+1\right)\right\}\right]^{(-1 / \beta)} \text {. }
$$

2.1.2. Median. Median of MF distribution is obtained by substituting $u=1 / 2$ in equation (13), that is,

$$
\text { Median }=\left[-\frac{1}{\alpha} \ln \left\{-\ln \left(\frac{1}{2}\left(e^{-1}+1\right)\right)\right\}\right]^{(-1 / \beta)} \text {. }
$$

2.1.3. Mode. Mode of MF is obtained by solving the following equation for $x$.

$$
\begin{aligned}
\frac{\mathrm{d}}{\mathrm{d} x} f_{\mathrm{MF}}(x) & =0, \quad \text { i.e. } \frac{\mathrm{d}}{\mathrm{d} x}\left(\frac{\alpha \beta x^{-(\beta+1)} e^{-\alpha x^{-\beta}-e^{-\alpha x^{-\beta}}}}{\left(1-e^{-1}\right)}\right)=0, \\
x^{-\beta}\left(e^{-\alpha x^{-\beta}}-1\right) & =\frac{-(\beta+1)}{\alpha \beta} .
\end{aligned}
$$

Mode of the distribution satisfies the above equation.

2.2. $r^{\text {th }}$ Moment of MF Distribution. Let $X \sim \operatorname{MF}(\alpha, \beta)$; and the $r^{\text {th }}$ moment of $X$ is as follows:

$$
\mu_{r} /=E\left(X^{r}\right)=\int_{0}^{\infty} x^{r} \frac{\alpha \beta x^{-(\beta+1)} e^{-\alpha x^{-\beta}-e^{-\alpha x}-\beta}}{\left(1-e^{-1}\right)} \mathrm{d} x .
$$

Using $x^{-\beta}=y$ and then $e^{-\alpha y}=z$ in (16), the expression will take the following form:

$$
\mu_{r} /=E\left(X^{r}\right)=-\frac{(-1 / \alpha)^{m}}{\left(1-e^{-1}\right)} \int_{0}^{1}(\ln z)^{m} e^{-z} \mathrm{~d} z,
$$

where $m=(-r / \beta)$.

Again substituting $\log z=u$ in (17) and after some simplification, the expression becomes 


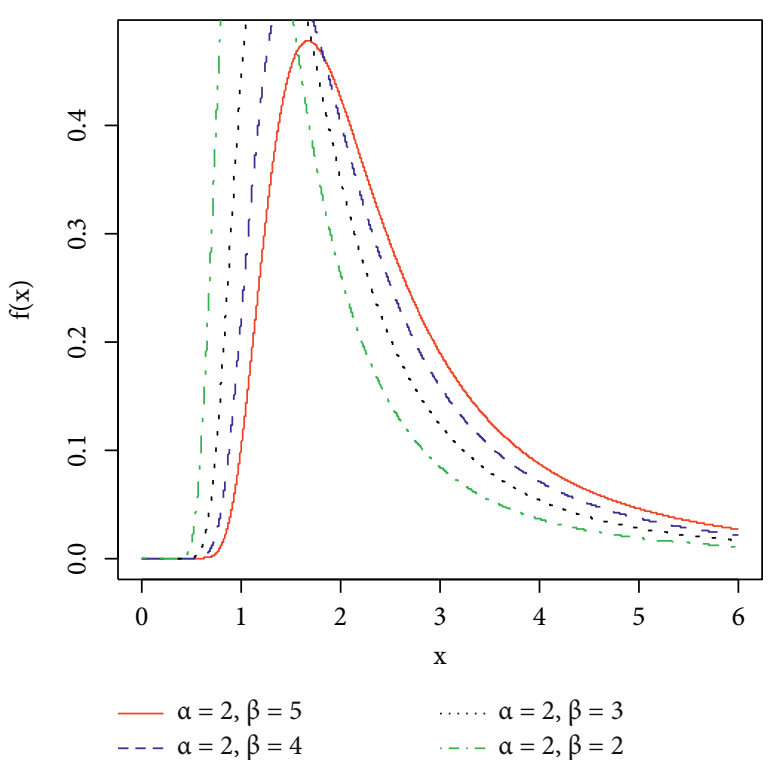

(a)

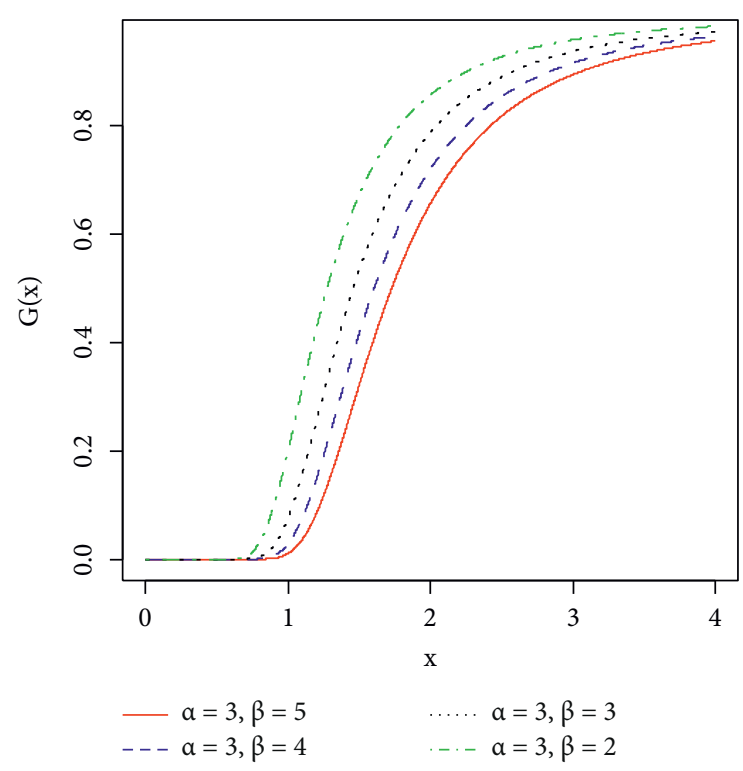

(b)

Figure 1: Graph of CDF and PDF of the MF model.



(a)

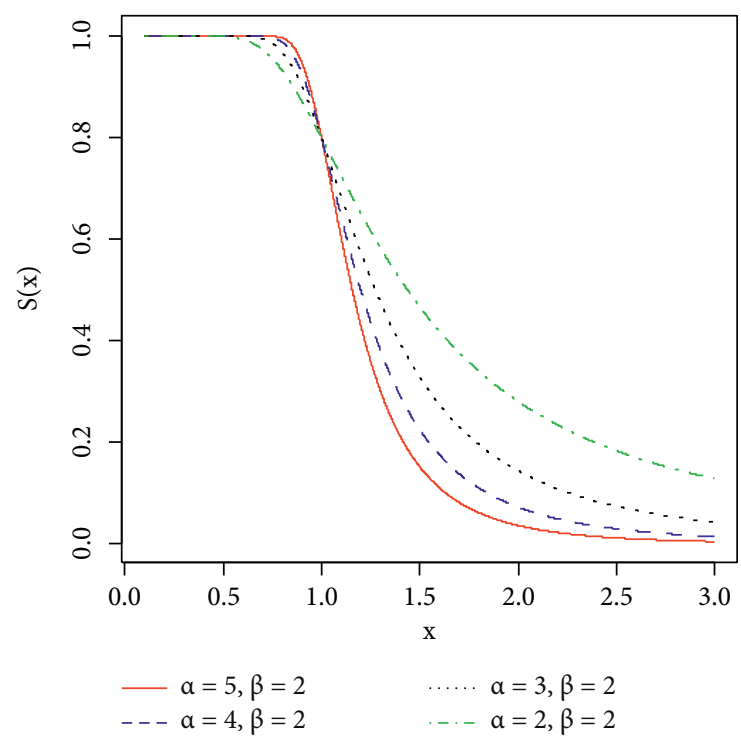

(b)

FIGURE 2: Graph of survival function and hazard function of MF distribution. 


$$
\mu_{r} /=E\left(X^{r}\right)=\frac{(-1 / \alpha)^{m}}{\left(e^{-1}-1\right)} \int_{-\infty}^{0} u^{m} e^{-e^{u}} e^{u} \mathrm{~d} z
$$

Using series notation $e^{-e^{u}}=\sum_{r=0}^{\infty}\left(\left(-e^{u}\right)^{k} / k !\right)$ in (18), we obtain

$$
\begin{aligned}
& \mu_{r} /=E\left(X^{r}\right)=\frac{(-1 / \alpha)^{m}}{\left(e^{-1}-1\right)} \sum_{k=0}^{\infty} \frac{(-1)^{k}}{k !} \lim _{b \longrightarrow-\infty}-\text { it } \int_{b}^{0} u^{m} e^{u(k+1)} \mathrm{d} z, \\
& \mu_{r} /=E\left(X^{r}\right)=\frac{(-1 / \alpha)^{m}}{\left(e^{-1}-1\right)} \sum_{k=0}^{\infty} \frac{(-1)^{k}}{k !} \lim _{b \longrightarrow-\infty}-\mathrm{it}\left[\frac{\Gamma(m+1,-b k-b)-\Gamma(m+1,0)}{(-k-1)^{m}(k+1)}\right],
\end{aligned}
$$

where $b>0$.

$$
M_{x}(t)=E\left(e^{t x}\right)=\int_{0}^{\infty} e^{t x} \frac{\alpha \beta x^{-(\beta+1)} e^{-\alpha x^{-\beta}-e^{-\alpha x}}}{\left(1-e^{-1}\right)} \mathrm{d} x .
$$

2.3. Moment Generating Function. Let $X \sim \operatorname{MF}(\alpha, \beta)$; then, the moment generating function is given by
Using series $e^{t x}=\sum_{r=0}^{\infty}\left(t^{r} x^{r} / r !\right)$ in (20) and simplifying, we have

$$
M_{x}(t)=\frac{(-1 / \alpha)^{m}}{\left(e^{-1}-1\right)} \sum_{k=0}^{\infty} \sum_{r=0}^{\infty} \frac{t^{r}(-1)^{k}}{r ! k !} \lim _{b \longrightarrow-\infty}\left[\frac{\Gamma(m+1,-b k-b)-\Gamma(m+1,0)}{(-k-1)^{m}(k+1)}\right],
$$

where $m=(-r / \beta)$ and $b>0$.

$$
f_{i: n}(x)=\frac{n !}{(i-1) !(n-i) !} f(x)[F(x)]^{i-1}[1-F(x)]^{(n-i)} .
$$

2.4. Order Statistics. Let $X_{1}, X_{2}, \ldots, X_{n}$ be a random sample taken from MF distribution, and let $X_{(1)} \leq X_{(2)} \leq \cdots \leq X_{(n)}$ denote the order statistics. Then, the probability density function of $X_{i: n}$ is given by
Substitute PDF and CDF of MF in equation (22), and we obtain distribution of $i^{\text {th }}$ order statistic as

$$
\begin{aligned}
& f_{i: n}(x)=\frac{n !}{(i-1) !(n-i) !}\left(\frac{\alpha \beta x^{-(\beta+1)} e^{-\alpha x^{-\beta}-e^{-\alpha x}-\beta}}{\left(1-e^{-1}\right)}\right)\left[\frac{e^{-\left(e^{-\alpha x}-\beta\right.}-1}{e^{-1}-1}\right]^{i-1}\left[1-\left(\frac{e^{-\left(e^{-\alpha x-\beta}\right)}-1}{e^{-1}-1}\right)\right]^{(n-i)}, \\
& f_{i: n}(x)=\frac{n !}{\left(1-e^{-1}\right)(i-1) !(n-i) !} \frac{1}{\left(e^{-1}-1\right)^{n-1}}\left[e^{-\left(e^{-\alpha x-\beta}\right)}-1\right]^{(i-1)} \alpha \beta x^{-(\beta+1)} e^{-\alpha x^{-\beta}-e^{-\alpha x}-\beta}\left[e^{-1}-e^{-\left(e^{-\alpha x-\beta}\right)}\right]^{(n-i)} .
\end{aligned}
$$

Lemma 3. The Renyi entropy of $X \sim M F(\alpha, \beta)$ is given as

$$
\mathrm{RE}_{X}(v)=\frac{1}{(1-v)} \log \left[\frac{\alpha^{((1-v) / \beta)} \beta^{\nu-1}(-1)^{l}}{\left(1-e^{-1}\right)^{v}} \sum_{k=0}^{\infty} \frac{(-v)^{k}}{k !} \lim _{b \longrightarrow-\infty}-\mathrm{it}\left[\frac{\Gamma(l+1,-b v-b k+b)-\Gamma(l+1,0)}{(-v-k+1)^{l}(\nu+k-1)}\right]\right],
$$

where $l=((\beta+1)(\nu-1) / \beta)$ and $b>0$.

Proof. The Renyi entropy of MF is given by

$$
\mathrm{RE}_{X}(v)=\frac{1}{1-v} \log \left\{\int_{-\infty}^{+\infty} f(x)^{v} \mathrm{~d} x\right\}=\frac{1}{1-v} \log \left[\int_{0}^{\infty}\left(\frac{\alpha \beta x^{-(\beta+1)} e^{-\alpha x^{-\beta}-e^{-\alpha x-\beta}}}{\left(1-e^{-1}\right)}\right)^{v} \mathrm{~d} x\right]
$$


Put $e^{-\alpha x^{-\beta}}=y$ in (25); the expression will take the form

$$
\mathrm{RE}_{X}(v)=\frac{1}{(1-\nu)} \log \left[\frac{\beta^{\nu-1}}{\left(1-e^{-1}\right)^{\nu}} \alpha^{((1-\nu) / \beta)}(-1)^{l} \int_{0}^{1}(\ln y)^{l} y^{\nu-1} e^{-\nu y} \mathrm{~d} y\right]
$$

where $l=((\beta+1)(\nu-1) / \beta)$. Using series notation $e^{-v y}=$ $\sum_{k=0}^{\infty}\left((\nu)^{k}(-y)^{k} / k !\right)$ in (26), the expression will take the following form:

$$
\operatorname{RE}_{X}(v)=\frac{1}{(1-\nu)} \log \left[\frac{\alpha^{((1-v) / \beta)} \beta^{\nu-1}(-1)^{l}}{\left(1-e^{-1}\right)^{\nu}} \sum_{k=0}^{\infty} \frac{(-\nu)^{k}}{k !} \int_{0}^{1}(\ln y)^{l} y^{\nu-1+k} \mathrm{~d} y\right]
$$

Again substituting $\ln y=z$ in (27) and simplifying, we obtain

$$
\begin{aligned}
& \mathrm{RE}_{X}(v)=\frac{1}{(1-\nu)} \log \left[\frac{\alpha^{((1-v) / \beta)} \beta^{\nu-1}(-1)^{l}}{\left(1-e^{-1}\right)^{\nu}} \sum_{k=0}^{\infty} \frac{(-v)^{k}}{k !} \lim _{b \longrightarrow-\infty}-\mathrm{it} \int_{b}^{0} z^{l} e^{z(\nu-1+k)} \mathrm{d} z\right] \\
& \operatorname{RE}_{X}(v)=\frac{1}{(1-v)} \log \left[\frac{\alpha^{((1-v) / \beta)} \beta^{\nu-1}(-1)^{l}}{\left(1-e^{-1}\right)^{\nu}} \sum_{k=0}^{\infty} \frac{(-v)^{k}}{k !} \lim _{b \longrightarrow-\infty} \mathrm{it}\left[\frac{\Gamma(l+1,-b \nu-b k+b)-\Gamma(l+1,0)}{(-v-k+1)^{l}(\nu+k-1)}\right]\right] \text {, } \\
& \mu(t)=\frac{1}{P(X>t)} \int_{t}^{\infty} P(X>x) \mathrm{d} x, \quad t \geq 0, \\
& \mu(t)=\frac{1}{S(t)}\left(E(t)-\int_{0}^{t} x f(x) \mathrm{d} x\right)-t, \quad t \geq 0 .
\end{aligned}
$$

where $l=((\beta+1)(\nu-1) / \beta)$ and. $b>0$.

2.5. Mean Residual Life Function. Let $X$ be the lifetime of an object having MF distribution. The mean residual life function is the average remaining life span that a component has survived until time $t$. The mean residual life function,

Note that say, $\mu(t)$, has the following expression:

$$
\begin{aligned}
\int_{0}^{t} x f(x) \mathrm{d} x & =\frac{1}{\left(1-e^{-1}\right)}\left(\frac{-1}{\alpha}\right)^{l} \sum_{k=0}^{\infty} \frac{(-1)^{k}}{k !} \lim _{b \longrightarrow-\infty}-\mathrm{it}\left[\frac{\Gamma\left(l+1,\left((\alpha k+\alpha) / t^{\beta}\right)\right)\left(-\alpha / t^{\beta}\right)^{l}}{(k+1)\left((\alpha k+\alpha) / t^{\beta}\right)^{l}}-\frac{b^{l} \Gamma(l+1,(-b k-b))}{(-b k-b)^{l}(k+1)}\right] \\
E(t) & =\frac{\alpha^{-l}(-1)^{l}}{\left(1-e^{-1}\right)} \sum_{k=0}^{\infty} \frac{(-1)^{k}}{k !} \lim _{b \longrightarrow-\infty} \mathrm{it}\left[\frac{\Gamma(l+1,-b k-b)-\Gamma(l+1,0)}{(-k-1)^{l}(k+1)}\right]
\end{aligned}
$$

where $l=(-1 / \beta)$. Put equation (9), (30), and (31) in (29), and we obtain 


$$
\begin{aligned}
\mu(t)= & \frac{\left(e^{-1}-1\right)}{\left(e^{-1}-e^{-\left(e^{-\alpha x^{-\beta}}\right)}\right)} \frac{1}{\left(1-e^{-1}\right)}\left(\frac{-1}{\alpha}\right)^{l} \sum_{k=0}^{\infty} \frac{(-1)^{k}}{k !} \lim _{b \longrightarrow-\infty} * \text { it } * \\
& {\left[\left[\frac{\Gamma(l+1,-b k-b)-\Gamma(l+1,0)}{(-k-1)^{l}(k+1)}\right]-\left[\frac{\Gamma\left(l+1,\left((\alpha k+\alpha) / t^{\beta}\right)\right)\left(-\alpha / t^{\beta}\right)^{l}}{(k+1)\left((\alpha k+\alpha) / t^{\beta}\right)^{l}}-\frac{b^{l} \Gamma(l+1,(-b k-b))}{(-b k-b)^{l}(k+1)}\right]\right]-t . }
\end{aligned}
$$

This is the final expression of mean residual life function.

$$
R=\int_{-\infty}^{+\infty} f_{1}(x) F_{2}(x) \mathrm{d} x .
$$

2.6. Stress-Strength Parameter. Let $X_{1}$ and $X_{2}$ be two independently and identically distributed variables such that $X_{1} \sim \operatorname{MF}\left(\alpha_{1}, \beta\right)$ and $X_{2} \sim \operatorname{MF}\left(\alpha_{2}, \beta\right)$. Then, the stressstrength parameter is defined by
Using equation PDF and CDF of MF in the above expression, the stress-strength parameter is given as

$$
\begin{aligned}
& R=\int_{0}^{\infty}\left(\frac{\alpha \beta x^{-(\beta+1)} e^{-\alpha_{1} x^{-\beta}-e^{-\alpha_{1} x^{-\beta}}}}{\left(1-e^{-1}\right)}\right)\left(\frac{e^{-\left(e^{-\alpha_{2} x^{-\beta}}\right)}-1}{\left(e^{-1}-1\right)}\right) \mathrm{d} x, \\
& R=\frac{1}{\left(e^{-1}-1\right)\left(1-e^{-1}\right)} \int_{0}^{\infty} \alpha_{1} \beta x^{-\beta-1} e^{-\alpha_{1} x^{-\beta}-e^{-\alpha_{1} x^{-\beta}}} e^{-\left(e^{-\alpha_{2} x^{-\beta}}\right)} \mathrm{d} x-\frac{1}{\left(e^{-1}-1\right)} .
\end{aligned}
$$

Substituting $x^{-\beta}=y$ in (34), we obtain

$$
R=\frac{1}{\left(e^{-1}-1\right)\left(1-e^{-1}\right)} \int_{0}^{\infty} \alpha_{1} e^{-\alpha_{1} y} e^{-e^{-\alpha_{1} y}} e^{-\left(e^{-\alpha_{2} y}\right)} \mathrm{d} y-\frac{1}{\left(e^{-1}-1\right)} .
$$

Again putting $e^{-\alpha_{1} y}=z$ in (35), it will take the following form:

$$
R=\frac{1}{\left(e^{-1}-1\right)^{2}} \int_{0}^{1} e^{-z} e^{-\left(e^{-\alpha_{2}\left(-\ln z / \alpha_{1}\right)}\right)} \mathrm{d} z-\frac{1}{\left(e^{-1}-1\right)} .
$$

Using series representation $e^{-z}=\sum_{k=0}^{\infty}\left((-z)^{k} / k !\right)$ and $e^{-\left(e^{-\alpha_{2}\left(-\ln z / \alpha_{1}\right)}\right)}=\sum_{m=0}^{\infty}\left(\left(-e^{\left(\alpha_{2} \ln z / \alpha_{1}\right)}\right)^{m} / m !\right)$ in (36), we get the expression as follows:

$$
R=\frac{1}{\left(e^{-1}-1\right)^{2}} \sum_{k=0}^{\infty} \sum_{m=0}^{\infty} \frac{(-1)^{k+m}}{k ! m !} \int_{0}^{1} z^{k} e^{n \ln z} \mathrm{~d} z-\frac{1}{\left(e^{-1}-1\right)},
$$

where $n=m\left(\alpha_{2} / \alpha_{1}\right)$. Using series representation $e^{n \ln z}=\sum_{i=0}^{\infty}\left((n \ln z)^{i} / i !\right)$ in (52), after simplification, we get the following expression:

$$
R=\frac{1}{\left(e^{-1}-1\right)^{2}} \sum_{k=0}^{\infty} \sum_{m=0}^{\infty} \sum_{i=0}^{\infty} \frac{(-1)^{k+m} n^{i}}{k ! m ! i !} \int_{0}^{1} z^{k}(\ln z)^{i} \mathrm{~d} z-\frac{1}{\left(e^{-1}-1\right)} .
$$

Again substituting $\ln z=u$ in (38) and simplifying, we obtain

$$
\begin{aligned}
& R=\frac{1}{\left(e^{-1}-1\right)^{2}} \sum_{k=0}^{\infty} \sum_{m=0}^{\infty} \sum_{i=0}^{\infty} \frac{(-1)^{k+m} n^{i}}{k ! m ! i !} \int_{-\infty}^{0} u^{i} e^{u(k+1)} \mathrm{d} u-\frac{1}{\left(e^{-1}-1\right)}, \\
& R=\frac{1}{\left(e^{-1}-1\right)^{2}} \sum_{k=0}^{\infty} \sum_{m=0}^{\infty} \sum_{i=0}^{\infty} \frac{(-1)^{k+m} n^{i}}{k ! m ! i !} \lim _{b \longrightarrow-\infty}-\mathrm{it} \int_{b}^{0} u^{i} e^{u(k+1)} \mathrm{d} u-\frac{1}{\left(e^{-1}-1\right)}, \\
& R=\frac{1}{\left(e^{-1}-1\right)^{2}} \sum_{k=0}^{\infty} \sum_{m=0}^{\infty} \sum_{i=0}^{\infty} \frac{(-1)^{k+m} n^{i}}{k ! m ! i !} \lim _{b \longrightarrow-\infty}\left[\frac{\Gamma(i+1,-b k-b)-\Gamma(i+1)}{(-k-1)^{i}(k+1)}\right]-\frac{1}{\left(e^{-1}-1\right)},
\end{aligned}
$$


Lemma 4. The mean waiting time, say $\bar{\mu}(t)$, of $M F$ distribution is given by

$$
\bar{\mu}(t)=t+\frac{1}{\left(e^{-\left(e^{-\alpha t^{-\beta}}\right)}-1\right)}\left(\frac{-1}{\alpha}\right)^{l} \sum_{k=0}^{\infty} \frac{(-1)^{k}}{k !} \lim _{b \longrightarrow-\infty}-\mathrm{it}\left[\frac{\Gamma\left(l+1,\left((\alpha k+\alpha) / t^{\beta}\right)\right)\left(-\alpha / t^{\beta}\right)^{l}}{(k+1)\left((\alpha k+\alpha) / t^{\beta}\right)^{l}}-\frac{b^{l} \Gamma(l+1,(-b k-b))}{(-b k-b)^{l}(k+1)}\right] .
$$

Proof. By definition, the mean waiting time of MF distribution is

$$
\bar{\mu}(t)=t-\frac{1}{F(t)} \int_{0}^{t} x f(x) \mathrm{d} x .
$$

$$
\int_{0}^{t} x f(x) \mathrm{d} x=\frac{1}{\left(1-e^{-1}\right)}\left(\frac{-1}{\alpha}\right)^{l} \sum_{k=0}^{\infty} \frac{(-1)^{k}}{k !} \lim _{b \longrightarrow-\infty}-\mathrm{it}\left[\frac{\Gamma\left(l+1,\left((\alpha k+\alpha) / t^{\beta}\right)\right)\left(-\alpha / t^{\beta}\right)^{l}}{(k+1)\left((\alpha k+\alpha) / t^{\beta}\right)^{l}}-\frac{b^{l} \Gamma(l+1,(-b k-b))}{(-b k-b)^{l}(k+1)}\right]
$$

and $\left.F(t)=\left(\left(e^{-\left(e^{-\alpha t}-\beta\right.}\right)-1\right) /\left(e^{-1}-1\right)\right)$, in $(41)$, we obtain

$$
\bar{\mu}(t)=t+\frac{1}{\left(e^{-\left(e^{-\alpha t^{-\beta}}\right)}-1\right)}\left(\frac{-1}{\alpha}\right)^{l} \sum_{k=0}^{\infty} \frac{(-1)^{k}}{k !} \lim _{b \longrightarrow-\infty} \text { it }\left[\frac{\Gamma\left(l+1,\left((\alpha k+\alpha) / t^{\beta}\right)\right)\left(-\alpha / t^{\beta}\right)^{l}}{(k+1)\left((\alpha k+\alpha) / t^{\beta}\right)^{l}}-\frac{b^{l} \Gamma(l+1,(-b k-b))}{(-b k-b)^{l}(k+1)}\right]
$$

2.7. Parameters' Estimation. Let $X_{1}, X_{2}, \ldots, X_{n}$ be a random sample of size $n$ selected from $\operatorname{MF}(\alpha, \beta)$; then, the loglikelihood function of MF distribution is given as

$$
\ln l(\alpha, \beta)=n \ln (\alpha \beta)-n \ln \left(1-e^{-1}\right)-\beta \sum_{i=1}^{n} \log x_{i}-\sum_{i=1}^{n} \log x_{i}-\alpha \sum_{i=1}^{n} x_{i}^{-\beta}-e^{-\alpha \sum_{i=1}^{n} x_{i}^{-\beta}}
$$

Differentiating equation (44) with respect to $\alpha$ and $\beta$ and equating them to 0 , we obtain

$$
\begin{aligned}
& \frac{\partial \ln l(\alpha, \beta)}{\partial \alpha}=\frac{n}{\alpha}-\sum_{i=1}^{n} x_{i}^{-\beta}+e^{-\alpha \sum_{i=1}^{n} x_{i}^{-\beta}} \sum_{i=1}^{n} x_{i}^{-\beta}=0 \\
& \frac{\partial \ln l(\alpha, \beta)}{\partial \beta}=\frac{n}{\beta}-\sum_{i=1}^{n} \log x_{i}-\alpha \sum_{i=1}^{n} x_{i}^{-\beta} \log x_{i}+\alpha e^{-\alpha \sum_{i=1}^{n} x_{i}^{-\beta}} \sum_{i=1}^{n} x_{i}^{-\beta} \log x_{i}=0 .
\end{aligned}
$$

Solving (45) and (46) together, we get the estimates of $\alpha$ and $\beta$. The Newton-Raphson method or the bisection method is used to get solution of the above equations as an analytical solution which is not possible. The maximum likelihood estimators (MLE) are asymptotically normally distributed, that is, $\sqrt{n}(\widehat{\alpha}-\alpha, \widehat{\beta}-\beta) \sim N_{2}(0, \Sigma)$, where $\Sigma$ is variance covariance matrix and can be obtained by inverting the observed Fisher information matrix $F$ given below: 


$$
F=\left(\begin{array}{c}
\frac{\partial^{2} \log l}{\partial \alpha^{2}} \frac{\partial^{2} \log l}{\partial \alpha \partial \beta} \\
\frac{\partial^{2} \log l}{\partial \alpha \partial \beta} \frac{\partial^{2} \log l}{\partial \beta^{2}}
\end{array}\right) .
$$

The second derivative of equations (45) and (46) with respect to $\alpha$ and $\beta$ yields (48)-(50) given as

$$
\begin{aligned}
& \frac{\partial^{2} \ln l}{\partial \alpha^{2}}=-\frac{n}{\alpha^{2}}-e^{-\alpha \sum_{i=1}^{n} x_{i}^{-\beta}}\left(\sum_{i=1}^{n} x_{i}^{-\beta}\right)^{2}, \\
& \frac{\partial^{2} \ln l}{\partial \beta^{2}}=-\frac{n}{\beta^{2}}-\alpha \sum_{i=1}^{n} x_{i}^{-\beta} \log x_{i}\left[\log x_{i}+\alpha e^{-\alpha \sum_{i=1}^{n} x_{i}^{-\beta}}-e^{-\alpha \sum_{i=1}^{n} x_{i}^{-\beta}} \log x_{i}\right],
\end{aligned}
$$

and

$$
\frac{\partial^{2} \ln l}{\partial \alpha \partial \beta}=\sum_{i=1}^{n} x_{i}^{-\beta} \log x_{i}^{-\beta}\left[e^{-\alpha \sum_{i=1}^{n} x_{i}^{-\beta}}-\alpha e^{-\alpha \sum_{i=1}^{n} x_{i}^{-\beta}}-1\right] .
$$

Asymptotic $(1-\zeta) 100 \%$ confidence intervals of the parameters of the proposed distribution can be obtained as

$$
\begin{aligned}
& \widehat{\alpha} \pm Z_{\zeta / 2} \sqrt{\Sigma_{11}}, \\
& \widehat{\beta} \pm Z_{\zeta / 2} \sqrt{\Sigma_{22}},
\end{aligned}
$$

where $Z_{\zeta}$ is the upper $\zeta^{\text {th }}$ percentile of the standard normal distribution.

\section{Simulations' Studies}

In order to measure the performance of MLE of the parameters of MF distribution, their mean square error (MSE) and bias are calculated using simulation study. We consider $W=100$ samples of sizes $n=50,70$, and 100 generated from the MF model. Bias and MSE are calculated using the expressions:

$$
\begin{aligned}
\text { Bias } & =\frac{1}{W} \sum_{1=1}^{w}\left(\widehat{b}_{i}-b\right), \\
\text { MSE } & =\frac{1}{W} \sum_{1=1}^{w}\left(\widehat{b}_{i}-b\right)^{2}, \quad \text { where } b=(\alpha, \beta) .
\end{aligned}
$$

Simulation results have been obtained for different values of $\alpha$ and $\beta$. The MSEs and bias are presented in Table 1 . The consistency behavior of MLE can be easily verified from these results as the MSEs and bias of the estimates decrease for all parameter combinations with increasing sample size. Hence, we conclude that MLE procedure executes very well in estimating the parameters of MF distribution.

\section{Applications}

Two practical datasets are used to assess the performance of MF distribution compared to Frechet distribution (FD), exponential distribution (ED), Weibull distribution (WD), alpha power inverse Weibull distribution (APIWD) [34], alpha power Weibull distribution (APWD) [35], and Kumaraswamy inverse Weibull distribution (KIWD) [36].

4.1. Dataset 1. The performance of the suggested model is assessed using two datasets. The first dataset is taken from the work of Gross and Clark [37] which consists of 20 observations of patients receiving an analgesic and is given as follows:

$$
\begin{array}{llllllllllllllllllll}
1.1 & 1.4 & 1.3 & 1.7 & 1.9 & 1.8 & 1.6 & 2.2 & 1.7 & 2.7 & 4.1 & 1.8 & 1.5 & 1.2 & 1.4 & 3.0 & 1.7 & 2.3 & 1.6 & 2.0 \text {. }
\end{array}
$$

4.2. Dataset 2. The second dataset consists of 40 wind-related catastrophes used by Hogg and klugman [38]. It includes claims of $\$ 2,000,000$. The sorted values, observed in millions, are as follows: 
TABLE 1: MSE and bias of MLE.

\begin{tabular}{|c|c|c|c|c|c|}
\hline Parameters & $N$ & $\operatorname{MSE}(\widehat{\alpha})$ & $\operatorname{MSE}(\widehat{\beta})$ & Bias $(\widehat{\alpha})$ & $\operatorname{Bias}(\widehat{\beta})$ \\
\hline \multirow{3}{*}{$\begin{array}{l}\alpha=5.557197 \\
\beta=7.558804\end{array}$} & 50 & 2.067219 & 0.9272915 & 0.5047117 & 0.2639728 \\
\hline & 70 & 0.9708544 & 0.7031859 & 0.3088091 & 0.2101214 \\
\hline & 100 & 0.7753223 & 0.372835 & 0.2578149 & 0.1197992 \\
\hline \multirow{3}{*}{$\begin{array}{l}\alpha=6.457197 \\
\beta=3.558804\end{array}$} & 50 & 2.026492 & 0.2259845 & 0.4415551 & 0.1637077 \\
\hline & 70 & 1.550174 & 0.1505096 & 0.4327197 & 0.1085847 \\
\hline & 100 & 0.8022496 & 0.1029645 & 0.1910671 & 0.03691258 \\
\hline \multirow{3}{*}{$\begin{array}{l}\alpha=5.457197 \\
\beta=3.558804\end{array}$} & 50 & 1.190428 & 0.2059653 & 0.4247818 & 2.342903 \\
\hline & 70 & 0.8619716 & 0.1373483 & 0.2461313 & 2.341802 \\
\hline & 100 & 0.4419579 & 0.08574337 & 0.04261996 & 2.333286 \\
\hline \multirow{3}{*}{$\begin{array}{l}\alpha=6.457197 \\
\beta=2.558804\end{array}$} & 50 & 2.518832 & 0.09526091 & 0.7185321 & 0.1320379 \\
\hline & 70 & 1.728531 & 0.09429495 & 0.4747864 & 0.07025322 \\
\hline & 100 & 0.8006676 & 0.04351014 & 0.1573213 & 0.01418112 \\
\hline \multirow{3}{*}{$\begin{array}{l}\alpha=4.557197 \\
\beta=4.558804\end{array}$} & 50 & 0.9564051 & 0.2857103 & 0.2557071 & 0.1324175 \\
\hline & 70 & 0.3593258 & 0.2089236 & 0.08780059 & 0.04002267 \\
\hline & 100 & 0.2750382 & 0.14968 & 0.02739781 & 0.01301724 \\
\hline \multirow{3}{*}{$\begin{array}{l}\alpha=6.557197 \\
\beta=1.558804\end{array}$} & 50 & 3.244032 & 0.04392251 & 0.5185741 & 0.03279788 \\
\hline & 70 & 1.495698 & 0.02470381 & 0.3520218 & 0.02578572 \\
\hline & 100 & 0.9790736 & 0.01922724 & 0.09188152 & 0.02093735 \\
\hline \multirow{3}{*}{$\begin{array}{l}\alpha=5.557197 \\
\beta=7.558804\end{array}$} & 50 & 2.067219 & 0.9272915 & 0.5047117 & 0.2639728 \\
\hline & 70 & 0.9708544 & 0.7031859 & 0.3088091 & 0.2101214 \\
\hline & 100 & 0.7753223 & 0.372835 & 0.2578149 & 0.1197992 \\
\hline \multirow{3}{*}{$\begin{array}{l}\alpha=2.557197 \\
\beta=7.558804\end{array}$} & 50 & 0.1693338 & 1.094077 & 0.1063671 & 0.3148875 \\
\hline & 70 & 0.08930482 & 0.4333755 & 0.08538295 & 0.1227512 \\
\hline & 100 & 0.06259663 & 0.3701105 & 0.02511625 & 0.03947369 \\
\hline
\end{tabular}

TABLE 2: Goodness of fit results for dataset 1.

\begin{tabular}{|c|c|c|c|c|c|c|c|c|c|c|}
\hline Distribution & \multicolumn{4}{|c|}{ MLE of the parameters } & AIC & CAIC & $\mathrm{BIC}$ & HQIC & $\mathrm{K}-\mathrm{S}$ & $P$ value \\
\hline MF & 6.4571 & 3.5588 & & & 34.88 & 35.59 & 36.87 & 35.27 & 0.107 & 0.9759 \\
\hline FD & 2.2255 & & & & 59.16 & 59.39 & 60.16 & 59.36 & 0.473 & 0.0003 \\
\hline ED & 0.5263 & & & & 67.67 & 67.89 & 68.66 & 67.86 & 0.439 & 0.0009 \\
\hline WD & 2.7843 & 2.1271 & & & 45.17 & 45.87 & 47.16 & 45.56 & 0.183 & 0.5104 \\
\hline APIWD & 1.7688 & 4.1692 & 5.4473 & & 36.79 & 38.29 & 39.77 & 37.37 & 0.124 & 0.9644 \\
\hline APWD & 10.9388 & 2.0312 & 0.4230 & & 46.58 & 48.08 & 49.57 & 47.16 & 0.162 & 0.6678 \\
\hline KIWD & 1.5668 & 1.2318 & 3.7669 & 3.5843 & 38.80 & 41.47 & 42.78 & 39.58 & 0.134 & 0.9540 \\
\hline
\end{tabular}

The bold values indicate that the proposed distribution is more significant as compared to other existing distributions.

TABLE 3: Goodness of fit results for dataset 2.

\begin{tabular}{lcccccccc}
\hline Distribution & \multicolumn{2}{c}{ MLE of the parameters } & AIC & CAIC & BIC & HQIC & K-S & $P$ value \\
\hline MF & $\mathbf{6 . 1 1 5 1}$ & $\mathbf{1 . 2 5 5 8}$ & $\mathbf{2 3 4 . 6 5}$ & $\mathbf{2 3 4 . 9 9}$ & $\mathbf{2 3 7 . 9 8}$ & $\mathbf{2 3 5 . 8 5}$ & $\mathbf{0 . 1 9 0}$ & $\mathbf{0 . 1 1 9 4}$ \\
FD & 0.7779 & & 280.98 & 281.09 & 282.64 & 281.58 & 0.558 & $5.61 e-11$ \\
ED & 0.1130 & & 250.03 & 250.15 & 251.70 & 250.64 & 0.202 & 0.08195 \\
WD & 1.0013 & 8.8585 & & 252.04 & 252.37 & 255.36 & 253.23 & 0.2017 \\
APIWD & 2.0394 & 1.4913 & 4.9249 & 237.33 & 238.01 & 242.32 & 239.12 & 0.2011 \\
APWD & 10.7622 & 0.69493 & 0.4071 & 255.18 & 255.86 & 260.17 & 256.97 & 0.2201 \\
\hline
\end{tabular}

The bold values indicate that the proposed distribution is more significant as compared to other existing distributions.

$\begin{array}{lllllllllllllllllllllllllllllllllllllllllll}2 & 2 & 2 & 2 & 2 & 2 & 2 & 2 & 2 & 2 & 2 & 2 & 3 & 3 & 3 & 4 & 4 & 4 & 5 & 5 & 5 & 6 & 6 & 6 & 6 & 8 & 8 & 9 & 15 & 17 & 22 & 23 & 24 & 24 & 25 & 27 & 32 & 43\end{array}$

In order to compare the MF model with other models, some standard model selection criteria such as Akaike's information criteria (AIC), consistent Akaike's information criteria (CAIC), Bayesian information criterion
(BIC), Hannan-Quinn information criteria (HQIC), Kolmogorov-Smirnov (K-S), and $P$ value are used. Tables 2 and 3 demonstrate results based on dataset 1 and 2, respectively. 

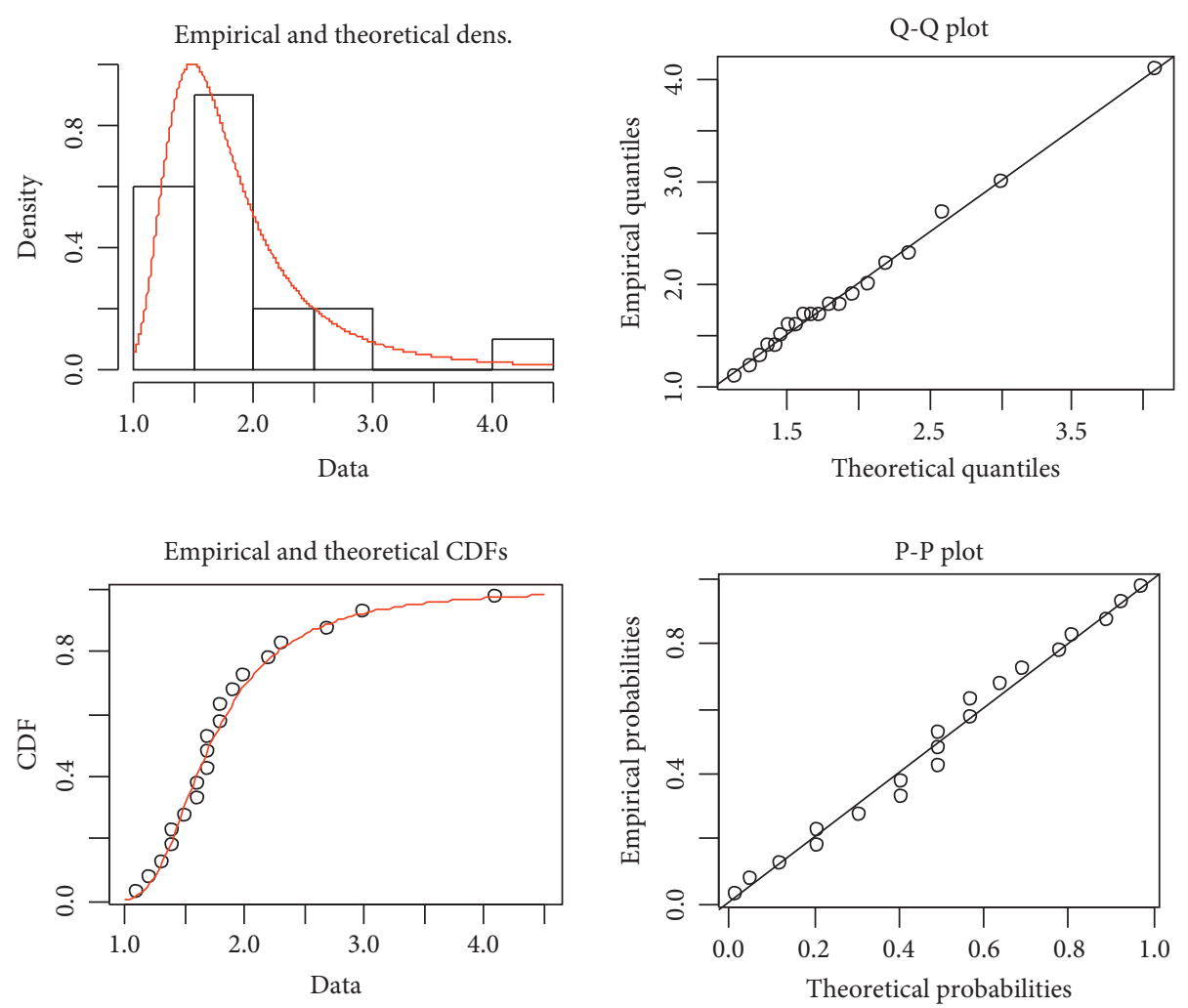

FIgURE 3: Plots of MF distribution for dataset 1.
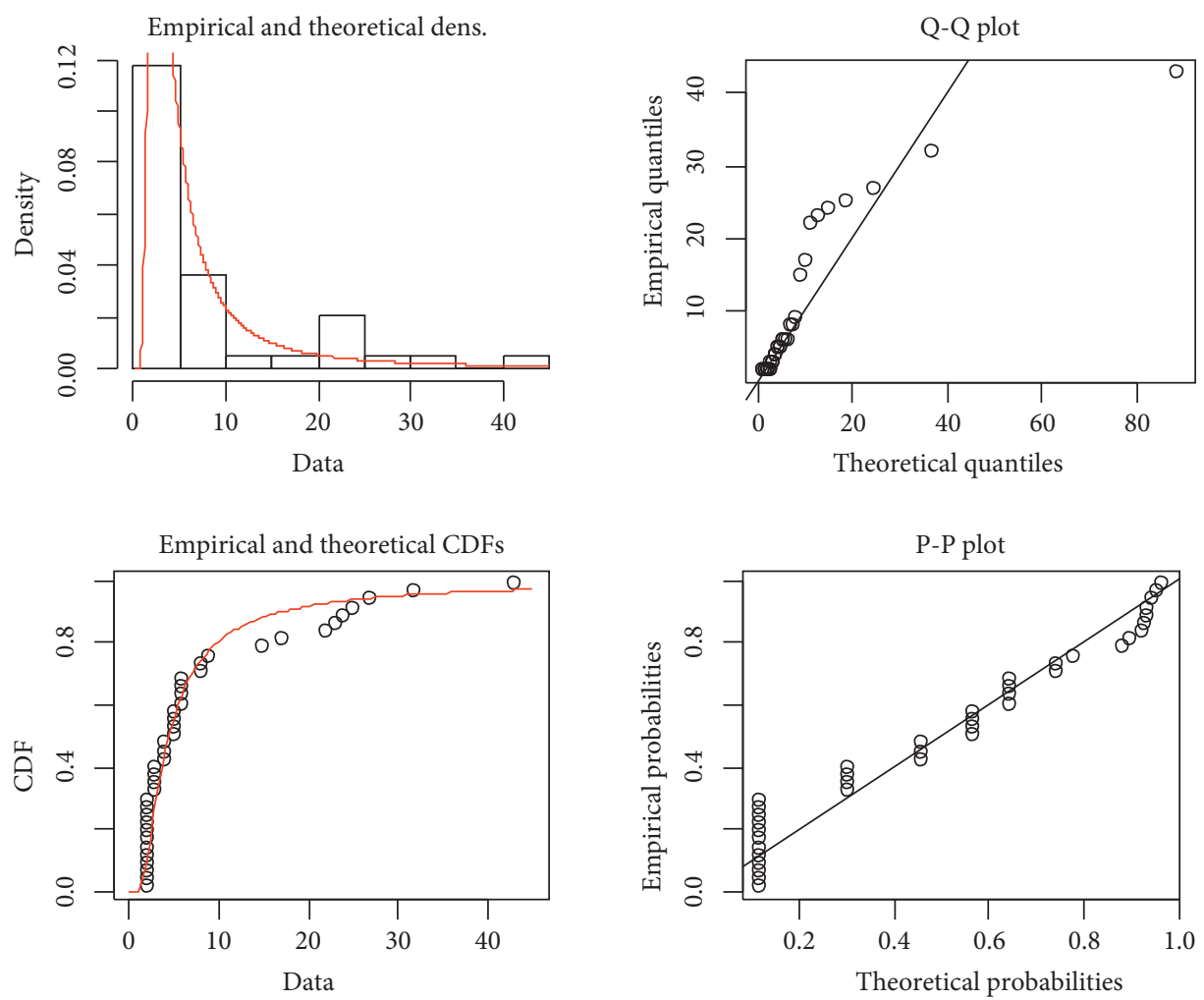

FIgURE 4: Plots of MF distribution for dataset 2. 
It is evident from the results in Tables 2 and 3 that the proposed MF distribution executes well as compared to other competitive distributions.

Figures 3 and 4 represent various graphs of MF distribution for dataset 1 and dataset 2 .

\section{Conclusion}

In this paper, a new method for deriving new continuous probability distributions has been offered which we called modified Frechet Class (MFC) of distributions. Also, a new probability model has been proposed using MFC. We called it modified Frechet (MF) distribution. Several statistical properties of the said distribution were derived and investigated for MF distribution. The MLE method was adopted to estimate the parameters of the proposed distribution. Simulation results showed that these estimates were consistent. In order to check the performance of the AFF model, two real datasets. The results based on these datasets revealed promising performance of the suggested model compared to some other distributions existing in the literature.

\section{Data Availability}

The data used in this paper are freely available upon citing this article.

\section{Conflicts of Interest}

The authors declare that they have no conflicts of interest to report regarding the present study.

\section{References}

[1] A. Azzalini, "A class of distributions which includes the normal ones," Scandinavian Journal of Statistics, vol. 12, no. 2 , pp. 171-178, 1985.

[2] G. S. Mudholkar and D. K. Srivastava, "Exponentiated weibull family for analyzing bathtub failure-rate data," IEEE Transactions on Reliability, vol. 42, no. 2, pp. 299-302, 1993.

[3] R. C. Gupta, P. L. Gupta, and R. D. Gupta, "Modeling failure time data by lehman alternatives," Communications in Statistics-Theory and Methods, vol. 27, no. 4, pp. 887-904, 1998.

[4] A. Marshall and I. Olkin, "A new method for adding a parameter to a family of distributions with application to the exponential and weibull families," Biometrika, vol. 84, no. 3, pp. 641-652, 1997.

[5] N. Eugene, C. Lee, and F. Famoye, "Beta-normal distribution and its applications," Communications in Statistics-Theory and Methods, vol. 31, no. 4, pp. 497-512, 2002.

[6] A. Alzaatreh, C. Lee, and F. Famoye, "A new method for generating families of continuous distributions," Metron, vol. 71, no. 1, pp. 63-79, 2013.

[7] M. A. Aljarrah, C. Lee, and F. Famoye, "On generating tx family of distributions using quantile functions," Journal of Statistical Distributions and Applications, vol. 1, no. 1, pp. 117, 2014.

[8] C. Lee, F. Famoye, and A. Y. Alzaatreh, "Methods for generating families of univariate continuous distributions in the recent decades," Wiley Interdisciplinary Reviews: Computational Statistics, vol. 5, no. 3, pp. 219-238, 2013.
[9] M. Jones, "On families of distributions with shape parameters," International Statistical Review, vol. 83, no. 2, 2014.

[10] R. Al-Aqtash, F. Famoye, and C. Lee, "On generating a new family of distributions using the logit function," Journal of Probability and Statistical Science, vol. 13, no. 1, pp. 135-152, 2015.

[11] A. Alzaatreh, F. Famoye, and C. Lee, "The gamma-normal distribution: properties and applications," Computational Statistics \& Data Analysis, vol. 69, pp. 67-80, 2014a.

[12] S. H. Abid and R. K. Abdulrazak, "Truncated frechet-G generator of distributions," Applied Mathematics, vol. 3, pp. 51-66, 2017.

[13] M. Ç. Korkmaz and A. İ. Genç, "A new generalized two-sided class of distributions with an emphasis on two-sided generalized normal distribution," Communications in StatisticsSimulation and Computation, vol. 46, no. 2, pp. 1441-1460, 2017.

[14] A. Alzaghal, F. Famoye, and C. Lee, "Exponentiated TX family of distributions with some applications," International Journal of Statistics and Probability, vol. 2, no. 3, p. 31, 2013.

[15] M. Aldeni, C. Lee, and F. Famoye, "Families of distributions arising from the quantile of generalized lambda distribution," Journal of Statistical Distributions and Applications, vol. 4, no. 1, p. 25, 2017.

[16] G. M. Cordeiro, M. Alizadeh, T. G. Ramires, and E. M. M. Ortega, "The generalized odd half-Cauchy family of distributions: properties and applications," Communications in Statistics-Theory and Methods, vol. 46, no. 11, pp. 56855705, 2017.

[17] A. Alzaatreh, C. Lee, F. Famoye, and I. Ghosh, "The generalized Cauchy family of distributions with applications," Journal of Statistical Distributions and Applications, vol. 3, no. 1, p. 12, 2016.

[18] M. A. Nasir, M. Aljarrah, F. Jamal, and M. H. Tahir, "A new generalized Burr family of distributions based on quantile function," Journal of Statistics Applications and Probability, vol. 6, no. 3, pp. 1-14, 2017.

[19] Z. J. Pe and Z. J. Jurek, "Operator-limit distributions in probability theory," Journal of the American Statistical Association, vol. 89, no. 427, 1994.

[20] K. Pearson, "Contributions to the mathematical theory of evolution. II. Skew variation in homogeneous material," Philosophical Transactions of the Royal Society of London-A, vol. 186, pp. 343-414, 1895.

[21] I. W. Burr, "Cumulative frequency functions," The Annals of Mathematical Statistics, vol. 13, pp. 215-232, 1942.

[22] M. Bourguignon, R. B. Silva, and G. M. Cordeiro, "The Weibull-G family of probability distributions," Journal of Data Science, vol. 12, pp. 53-68, 2014.

[23] M. Elgarhy, A. S. Hassan, and M. Rashed, "Garhy-generated family of distributions with application," Mathematical Theory and Modeling, vol. 6, pp. 1-15, 2016.

[24] G. M. Cordeiro and M. De Castro, "A new family of generalized distributions," Journal of Statistical Computation and Simulation, vol. 81, pp. 883-893, 2011.

[25] A. S. Hassan, M. Elgarhy, and M. Shakil, "Type II half Logistic family of distributions with applications," Pakistan Journal of Statistics and Operation Research, vol. 13, pp. 245-264, 2017.

[26] M. Elgarhy, M. Haq, G. Ozel, and N. Arslan, "A new exponentiated extended family of distributions with Applications," Gazi University Journal of Science, vol. 30, pp. 101-115, 2017.

[27] A. S. Hassan and M. Elgarhy, "Kumaraswamy Weibull-generated family of distributions with applications," Advances and Applications in Statistics, vol. 48, pp. 205-239, 2016. 
[28] A. S. Hassan and M. Elgarhy, "A New family of exponentiated Weibull-generated distributions," International Journal of Mathematics and Applications, vol. 4, pp. 135-148, 2016.

[29] M. Haq and M. Elgarhy, "The odd Frchet-G family of probability distributions," Journal of Statistics Applications \& Probability, vol. 7, pp. 185-201, 2018.

[30] A. M. Almarashi and M. Elgarhy, "A new muth generated family of distributions with applications," The Journal of Nonlinear Science and Applications, vol. 11, pp. 1171-1184, 2018.

[31] S. Kotz and D. Vicari, "Survey of developments in the theory of continuous skewed distributions," Metron, vol. 63, pp. 225-261, 2005.

[32] A. Mahdavi and D. Kundu, "A new method for generating distributions with an application to exponential distribution," Communications in Statistics-Theory and Methods, vol. 46, pp. 6543-6557, 2017.

[33] S. Boyd and L. Vendenberghe, Convex Optimization, Cambridge University Press, Cambridge, UK, 2004.

[34] D. A. Ramadan and A. W. Magdy, "On the alpha-power inverse weibull distribution," International Journal of Computer Applications, vol. 181, no. 11, pp. 6-12, 2018.

[35] M. Nassar, A. Alzaatreh, M. Mead, and A. Kasem, "Alpha power Weibull distribution :Properties and applications," Communications in Statistics-Theory and Methods, vol. 46, no. 20, pp. 10236-10252, 2017.

[36] M. Q. Shahbaz, S. Shahbaz, and N. S. Butt, "The kumaraswamy-inverse weibull distribution," Pakistan Journal of Statistics and Operation Research, vol. 8, no. 3, pp. 479-489, 2012.

[37] A. J. Gross and V. A. Clark, Survival Distributions: Reliability Applications in the Biomedical Sciences, John Wiley \& Sons, New York, NY, USA, 1975.

[38] R. Hogg and S. A. Klugman, Loss Distributions, Wiley, New York, NY, USA, 1984. 